\title{
Analysis of Aviation Ad-hoc Network Link Performance
}

\author{
Xiaoyan Huang \\ Chengdu University of Information Technology, Chengdu, 610225, China \\ Email: yyq@cuit.edu.cn
}

Keywords: MANET; Aviation Ad-hoc Network; Network Node

\begin{abstract}
Aviation ad-hoc network with the characteristics of large scale, high dynamics and the small node density, there are many problems exist in the high-speed mobile environment. The link constituted between high speed airplanes has a certain particularity. Consequently, the aviation ad-hoc network protocol design must first study the dynamic link. This paper adopted the method of probability calculation to deduce the mathematical formulas that represent the link life, expectations of the relative rate. Then the simulation experiments verified the accuracy of theoretical calculation.
\end{abstract}

\section{Introduction}

Aviation ad-hoc network is a MANET composed of civil aviation aircrafts in certain airspace. The aircrafts can be able to exchange their flight status and awareness information, and forward control command information send from the ground to each other, also can be connected to the ground INTERNET gateway in a single hop or multi-hop INTERNET gateway. Aviation ad-hoc network has the ability of self-organization, self-healing and fast, efficient networking ${ }^{[1]}$. It has a broad application prospect.

\section{Network modeling of link dynamic}

Link dynamics analysis is the foundation of the network protocol design, and the research has great significance for aviation ad-hoc network ${ }^{[2]}$. The measurements of link dynamic including link life, residual link life, new link formation rate and the link rate

Due to the flight speed and direction of flight is unchanged, the aviation ad-hoc network belongs to the quasi-linear MANET ${ }^{[3]}$. Random way points and random walk model is not applicable to aviation ad-hoc network, and constant velocity (CV) mobile model can accurately describe the movement rule of the civil aviation aircrafts ${ }^{[4]}$. Establish the rectangular coordinate system XOY as figure 2. Assume that node 1is static, node 2 move from A to the communication area of node 1 with relative speed and leave it.

$$
\begin{gathered}
v \stackrel{\Delta}{=}|v|=\sqrt{v_{1}^{2}+v_{2}^{2}-2 v_{1} v_{2} \cos \theta} \\
\phi \stackrel{\Delta}{=} \angle v=\arctan \left(\frac{\sin \theta}{\cos \theta-v_{1} / v_{2}}\right)
\end{gathered}
$$




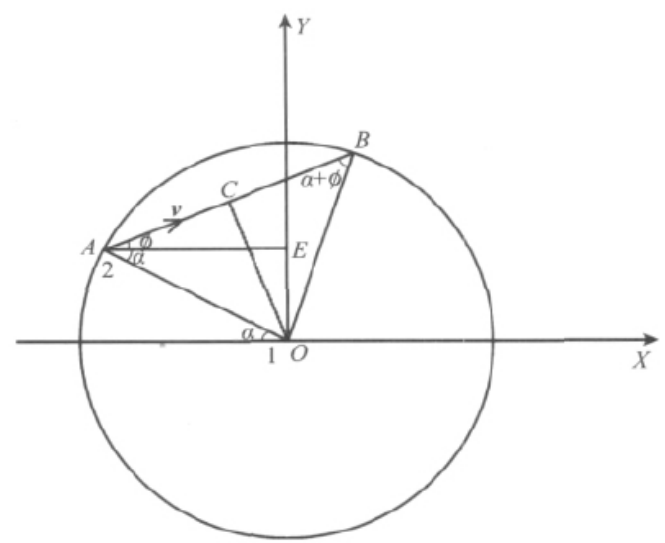

Figure 1 communication link of node 1 and node 2

the joint probability density function of random variables $\mathrm{v}, \phi$ and $\alpha$ is:

$f_{v \phi \alpha}(v, \phi, \alpha)=\frac{1}{4 \pi(b-a)} v \cos (\alpha+\phi) g\left(v, \phi, v_{1}\right) \cdot\left\{u\left(\alpha+\left(\frac{\pi}{2}+\phi\right)\right)-u\left(\alpha-\left(\frac{\pi}{2}-\phi\right)\right)\right\}$

$u(\cdot)$ is the standard unit step function.

$$
\begin{gathered}
g\left(v, \phi, v_{1}\right)=\frac{u\left(h\left(v, \phi, v_{1}\right)-\alpha\right)-u\left(h\left(v, \phi, v_{1}\right)-b\right)}{h\left(v, \phi, v_{1}\right)} \\
h\left(v, \phi, v_{1}\right)=\sqrt{v^{2}+v_{1}^{2}+2 v v_{1} \cos \phi}
\end{gathered}
$$

\section{3 . The analysis of the dynamic link}

(1) The relative velocity

In CV mobile model, the probability density function of the relative velocity $\mathrm{v}$ is:

$$
f_{v}(v)=\int_{a}^{b} \int_{-\pi}^{\pi} f_{v \phi}(v, \phi) d \phi d v_{1}=\int_{a}^{b} \int_{-\pi}^{\pi} \frac{1}{2 \pi(b-a)} v g\left(v, \phi, v_{1}\right) d \phi d v_{1}
$$

Use MATLAB to calculate formula (6). The result is shown in figure 2. The figure shows that the relative rate spread over $100 \sim 400 \mathrm{~m} / \mathrm{s}$, and $300 \sim 400 \mathrm{~m} / \mathrm{s}^{\text {are most concentrated }}{ }^{[5]}$.

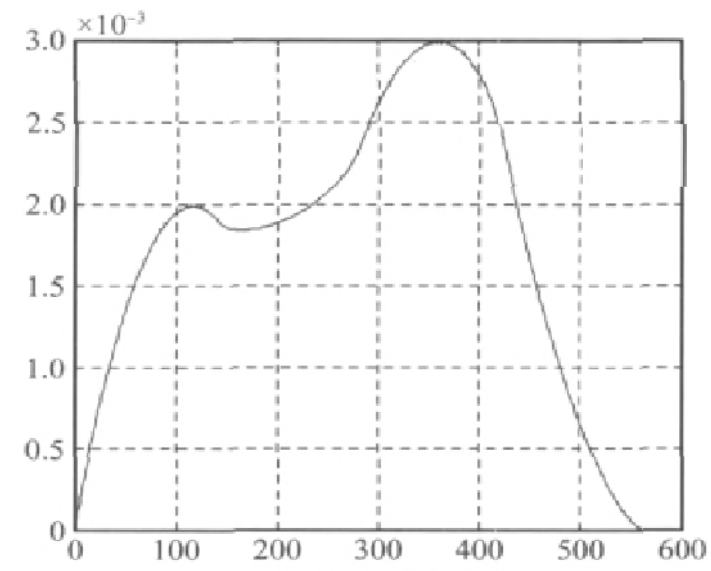

Figure 2 the probability density function of relative rate

(2) Link life

The link length of node 2 in the communication range of node 1 is $d_{\text {link }}=|2 R \cdot \cos (\alpha+\phi)|=2 R|\cos (\alpha+\phi)|$, so the link life is $t_{\text {link }}=\frac{|2 R \cdot \cos (\alpha+\phi)|}{v}$, and the link life expectancy is:

$$
T_{\text {link }}=E_{v, \phi, \alpha}\left[t_{\text {link }}\right]=\int_{0}^{\infty} \int_{-\pi}^{\pi} \int_{-\pi}^{\pi} t_{\text {link }} f_{v \phi \alpha}(v, \phi, \alpha) d \alpha d \phi d v
$$


Substituted into the formulas to calculate the final result:

$$
T_{\text {link }}=\frac{R}{2(b-a)}\left(\int_{0}^{\pi} \ln \left|\frac{b+\sqrt{b^{2}-v_{1}^{2} \sin ^{2} \phi}}{v_{1}+v_{1} \cos \phi}\right| d \phi-\int_{\phi_{0}}^{\pi} \ln \left|\frac{a+\sqrt{a^{2}-v_{1}^{2} \sin ^{2} \phi}}{a-\sqrt{a^{2}-v_{1}^{2} \sin ^{2} \phi}}\right| d \phi\right)
$$

In the formula $\phi_{0}=\pi-\arcsin \left(\frac{\alpha}{v_{1}}\right)$.

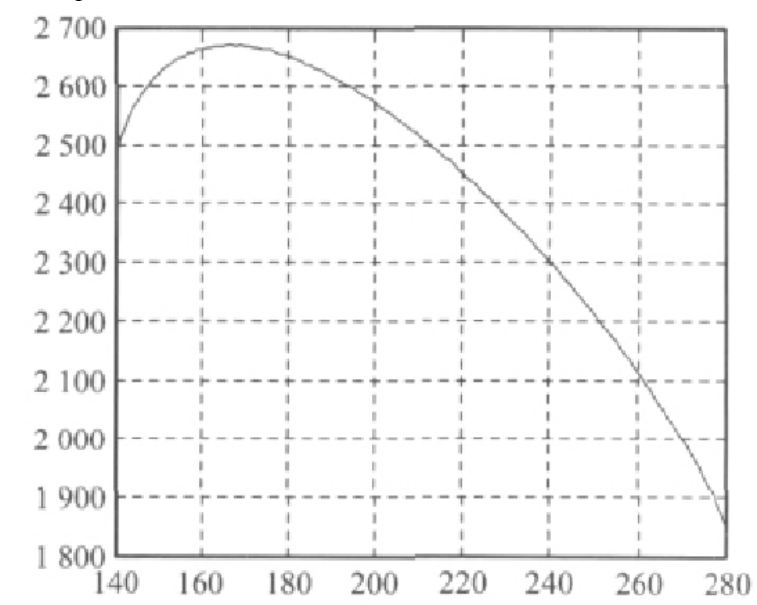

Figure 3 the relationship between link life expectancy and node rate

The numerical calculation results of formula (8) as shown in figure 3, it shows that the expectation of life is first increases then decreases with the node rate increases. Although in aviation ad-hoc network, the node movement rate is very high, because of the long communication distance, the link also can maintain a long time.

\section{Simulation results}

Build a network model in the MATLAB, set 100 nodes in the network scenario and the movement rate and direction is random. Set different movement rate for node 1and repeat the experiment.

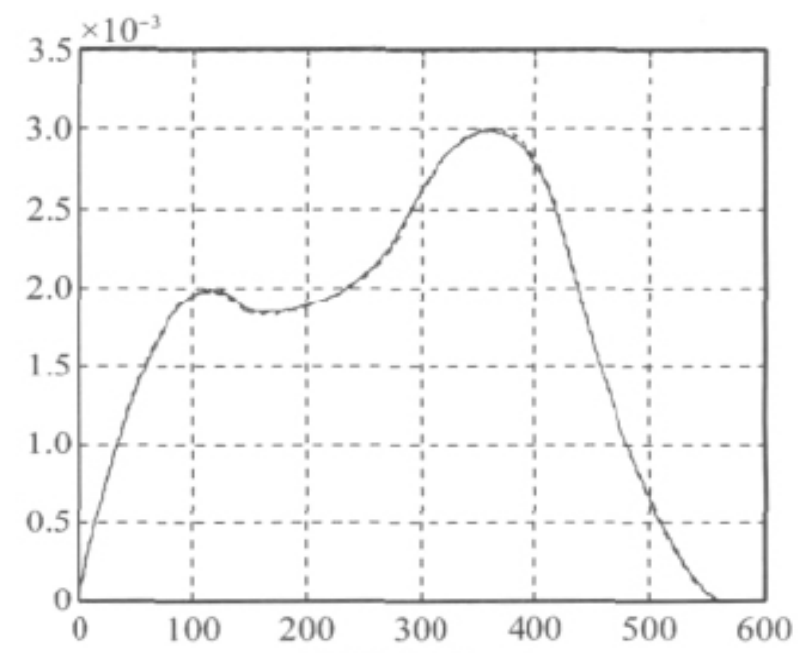

Figure 4 the theoretical value and simulation value of relative rate 


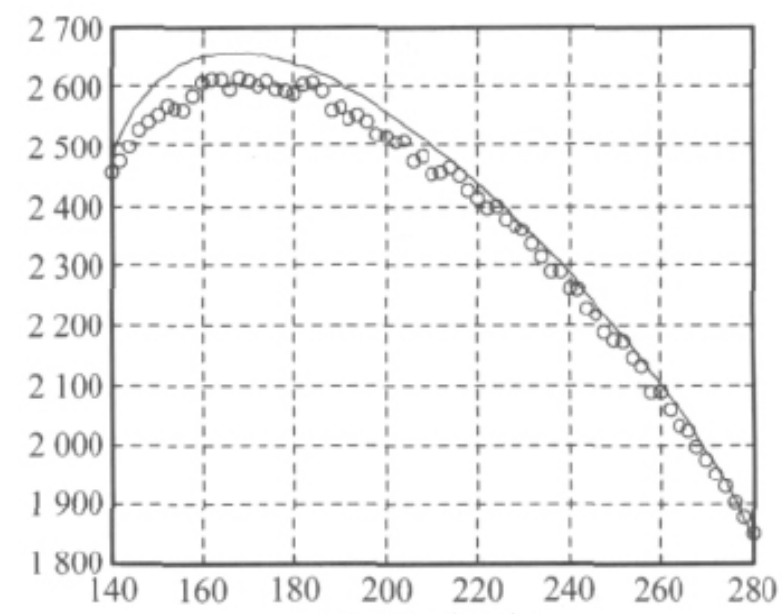

Figure 5 the theoretical value and simulation value of links life expectancy

\section{Conclusions}

This article adopted the method of probability calculation to study the link dynamics of aviation ad-hoc network. The mathematical model of links between airplanes is established. Use the theory of probability to deduce the mathematical formula of the relative velocity and link life expectancy, and verified by simulation experiments.

\section{References:}

[1] Ehssan Sakhaee, Abbas Jamalipour, Nei Kato. Aeronautical Ad Hoc Networks. Proc IEEE WCNC06. 2006

[2] Ehssan S,Abbas J, Nei K.Aeronautical Ad Hoc networks. IEEE WCNC. 2006

[3] S. Chakrabarti and A. Mishra. Quality of service challenges for wireless mobile ad hoc networks. 2004

[4] Yi S,Pei Y,Kalyanaraman S.On the capacity improvement of ad hoc wireless networks using directional antennas. Proceedings of ACM MobiHoc. 2003

[5] Kingsbury R W. Mobile Ad hoc networks for oceanic aircraft communications. . 2009 